\title{
RADIATION PROTECTION IN THE HEALTH SCIENCES Second Edition
}


This page intentionally left blank 


\title{
RADIATION PROTECTION IN THE HEALTH SCIENCES Second Edition
}

\author{
Marilyn E. Noz, Ph.D. \\ Professor of Radiology \\ New York University School of Medicine \\ New York, NY 10016, USA \\ and \\ Gerald Q. Maguire Jr., Ph.D. \\ Professor of Computer Communication Systems \\ Department of Communication Systems \\ School of Information and Communication Systems \\ Royal Institute of Technology \\ S-164 40 Kista, Sweden
}




\title{
Published by
}

World Scientific Publishing Co. Pte. Ltd.

5 Toh Tuck Link, Singapore 596224

USA office: 27 Warren Street, Suite 401-402, Hackensack, NJ 07601

UK office: 57 Shelton Street, Covent Garden, London WC2H 9HE

\author{
Library of Congress Cataloging-in-Publication Data \\ Noz, Marilyn E. \\ Radiation protection in the health sciences ( 2 nd ed.) : with problem solutions \\ manual / by Marilyn E. Noz \& Gerald Q. Maguire, Jr. \\ p. ; cm. \\ Includes bibliographical references and index. \\ ISBN-13 978-981-270-596-9 -- ISBN-10 981-270-596-1 \\ ISBN-13 978-981-270-597-6 (pbk) -- ISBN-10 981-270-597-X (pbk) \\ 1. Radiology, Medical--Safety measures. 2. Radiation Protection. I. Maguire, Gerald Q. \\ II. Title.
}

R895 .N688 2007

616.9'89705--dc22

2007011846

\section{British Library Cataloguing-in-Publication Data}

A catalogue record for this book is available from the British Library.

Copyright ( 2007 by World Scientific Publishing Co. Pte. Ltd.

All rights reserved. This book, or parts thereof, may not be reproduced in any form or by any means, electronic or mechanical, including photocopying, recording or any information storage and retrieval system now known or to be invented, without written permission from the Publisher.

For photocopying of material in this volume, please pay a copying fee through the Copyright Clearance Center, Inc., 222 Rosewood Drive, Danvers, MA 01923, USA. In this case permission to photocopy is not required from the publisher.

Printed in Singapore. 


\section{To our parents and to Judge and Mrs. Edwin M. Clark}


This page intentionally left blank 


\section{Preface}

The discovery of X-radiation (W. C. Röntgen) and the subsequent discovery of natural radioactivity (A.H. Becquerel) and isolation of individual radioactive elements, particularly radium (M. S. Curie), were followed immediately by the employment of ionizing radiation in medical practice for both diagnostic and therapeutic purposes. At first the risks associated with the use of ionizing radiation were not fully appreciated. As ionizing radiation was more frequently and widely employed, these risks were uncovered and evaluated.

Since the first quarter of the previous century protection from ionizing radiation has been an important concern. As the medical uses of ionizing radiation have increased, the importance of understanding risk versus benefit has also increased. As the twentieth century progressed, advances in nuclear physics led to new applications which could expose the human population to ionizing radiation, e.g., nuclear power plants coupled with spent fuel rod disposal and plant decommissioning; and the manufacture, deployment, use, and disposal of nuclear weapons. Additionally, public consciousness concerning the potential effects of ionizing radiation on the human population increased due to such events as the accident at the Three Mile Island and Chernobyl nuclear power plants, by the dismantling of nuclear weapons, and by the development of medical diagnostic techniques such as ultrasound and nuclear magnetic resonance imaging which are alternatives to procedures involving radiation. This has resulted in the radiologist, health or nuclear physicist, and radiologic technologist frequently being called upon as experts in evaluating risk and protection measures, as well as having to answer questions concerning absorbed dose and its effect on the human body. In addition, as individuals these professionals have a personal interest, as well as professional requirement, to consider radiation safety in their work.

The purpose of this book is to help the reader understand and respect the recommendations relating to the safe use of ionizing radiation. It is intended for courses in an academic or training program for all those who may encounter ionizing radiation. It is directed primarily toward students preparing for a career as a radiologic technologist, medical physicist, health physicist, or radiologist. The practicing physicist, physician, technologist, or nurse will find it a source of material regarding radiation protection standards and techniques. The tables and graphs shown throughout the book are only illustrative and are only to be used for study. For professional practice, the latest primary sources for data should be utilized. 
This book begins with a description of the fundamental processes by which ionizing radiation is produced and interacts with matter. It details the operation of radiation detection instruments and their use as survey and personnel monitors; includes the basic biologic effects of ionizing radiation along with the current units, regulations, and recommendations which govern exposure of radiation workers and the general public to ionization radiation; reviews the recommendations regarding the use of medical X-ray generators and radionuclides, and discusses good working habits associated with the use of such sources. This book also delineates the general and specific means used for protection against external ionizing radiation and explicates how to calculate absorbed dose from both internally deposited radionuclides and external sources of radiation. Each chapter contains review questions as well as problems. SI units are used extensively throughout the text as it is necessary to know and understand them. The appendices contain a discussion of units and logarithms. An extensive glossary is provided as well as and a bibliography which includes the relevant publications of national and international organizations. Answers to the problems are provided at the end of the book.

As with any book, the contents reflect criticisms and suggestions from numerous people. We would like to thank in particular the following: the many instructors and students who have used the two editions of the former book entitled Radiation Protection in the Radiologic and Health Sciences and the first edition of this book, our colleagues in the Department of Radiology at New York University, the Institute of Medical Radiation Physics at the Karolinska Institute and Stockholm University, and the Department of Communication Systems at the Royal Institute of Technology (KTH) in Stockholm, Sweden.

Marilyn E. Noz Gerald Q. Maguire, Jr.

December 2006 


\section{Contents}

Preface...$\ldots \ldots \ldots \ldots \ldots \ldots \ldots \ldots \ldots \ldots \ldots \ldots \ldots \ldots \ldots \ldots$ vii Chapter 1

Fundamental Concepts $\quad 1$

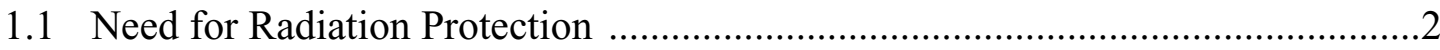

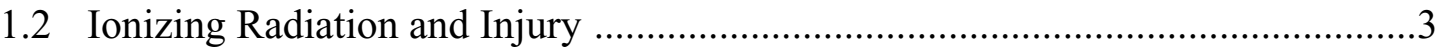

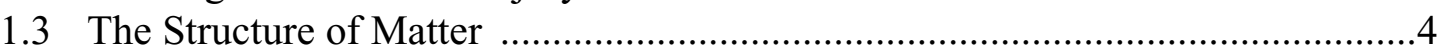

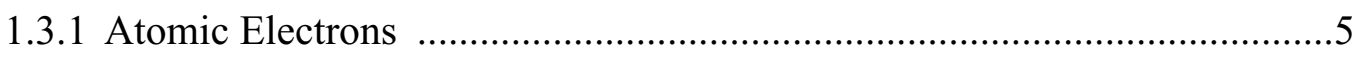

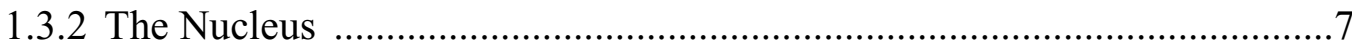

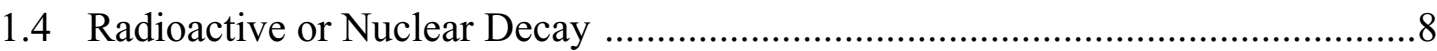

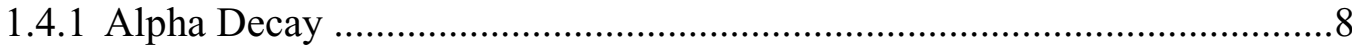

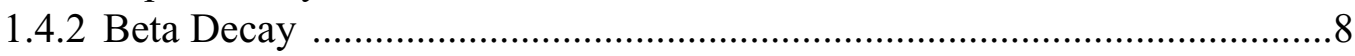

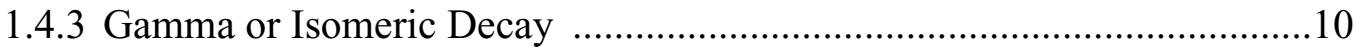

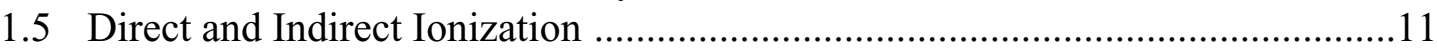

1.5.1 General Interaction Mechanisms ........................................................11

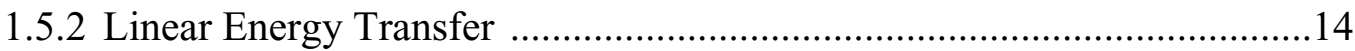

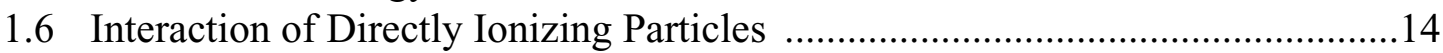

1.6.1 Alpha Particles .................................................................................

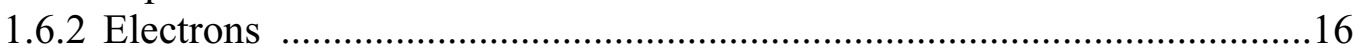

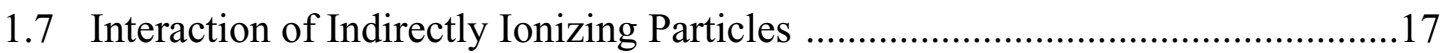

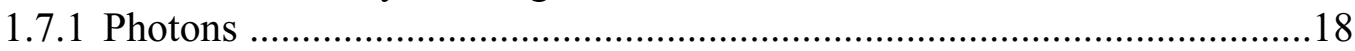

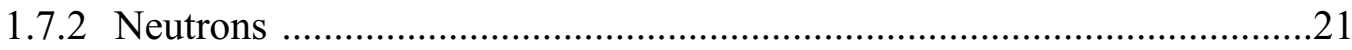

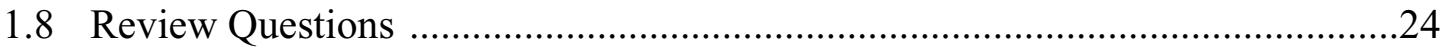

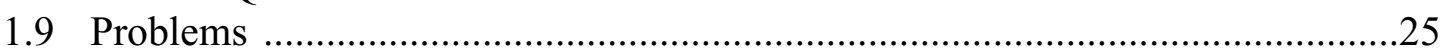

Chapter 2

$\begin{array}{lr}\text { Detectors } & \mathbf{2 7}\end{array}$

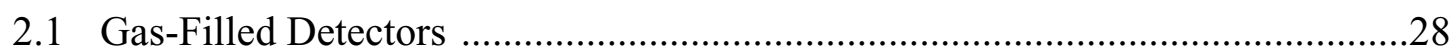

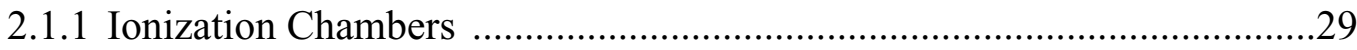

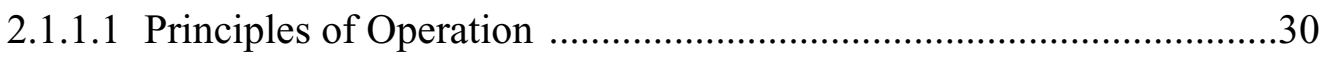

2.1.1.2 Electron Movement to Collector ………….......................................30

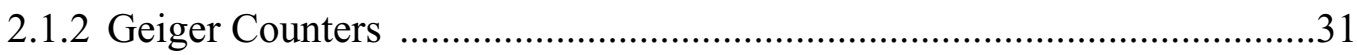

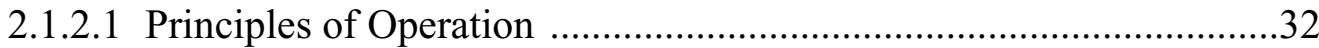

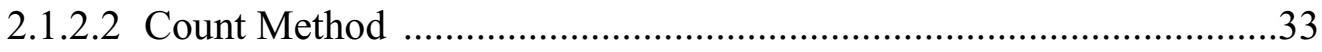

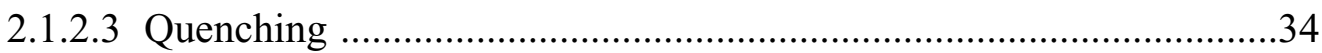

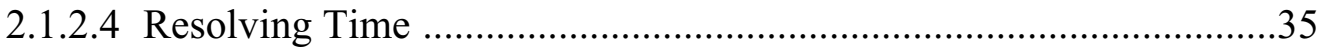

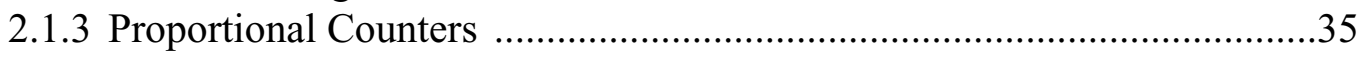

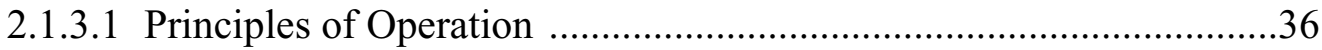

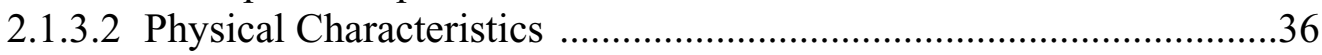

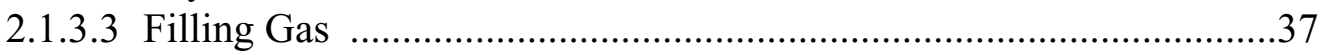

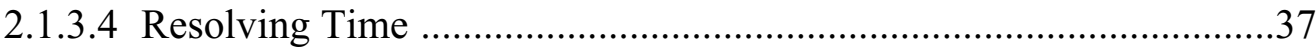

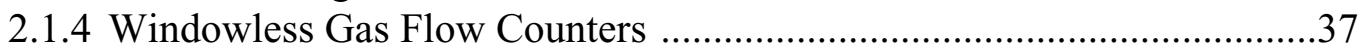




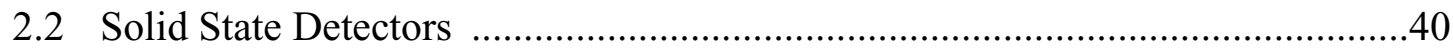

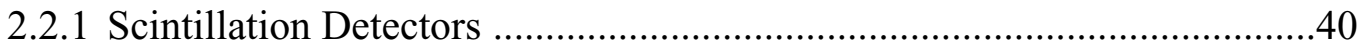

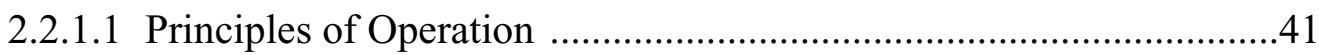

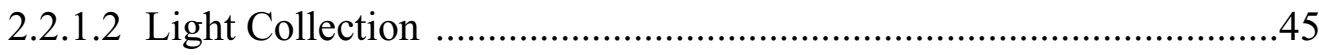

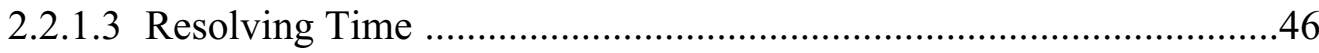

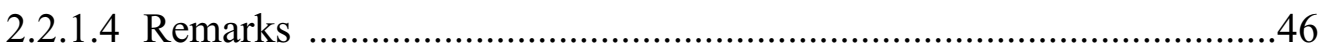

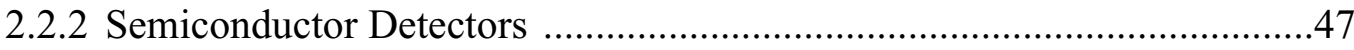

2.2.2.1 Principles of Operation ……...........................................................47

2.2.2.2 Types of Junction Semiconductors Detectors ...................................49

2.2.2.3 Operating Characteristics of Junction Detectors .................................53

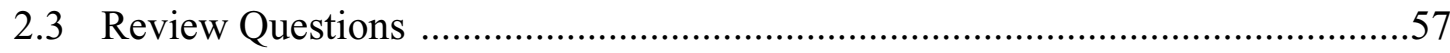

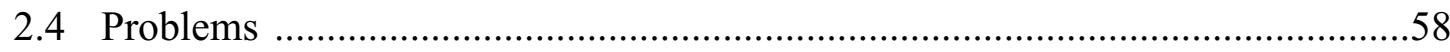

Chapter 3

Units Associated with Radiation Protection 59

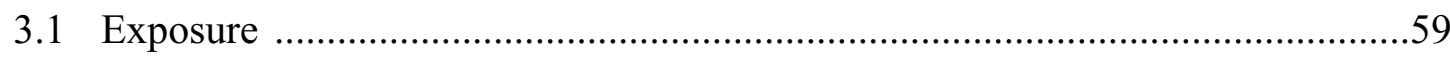

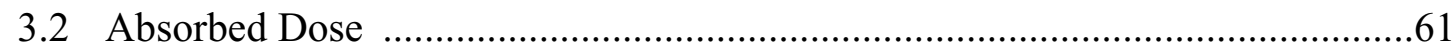

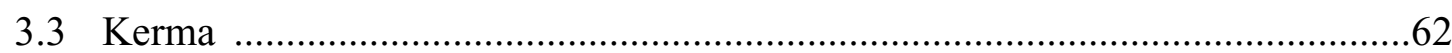

3.4 Relative Biological Effectiveness and Radiation Protection ............................63

3.5 Equivalent Dose and Effective Dose .................................................................65

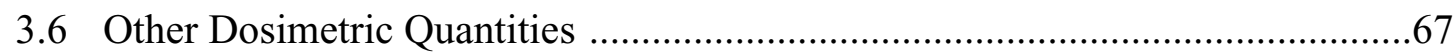

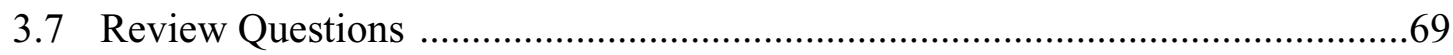

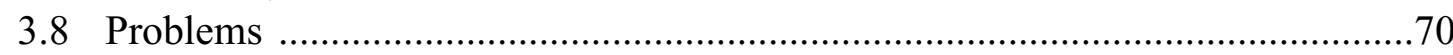

Chapter 4

Biological Effects and Effective/Equivalent Dose Limits $\quad 71$

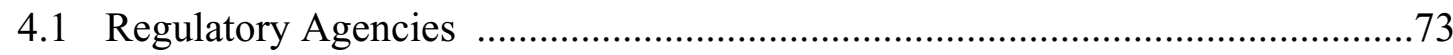

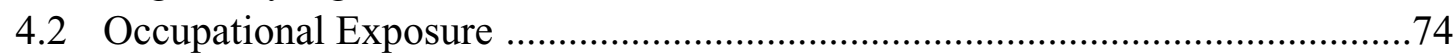

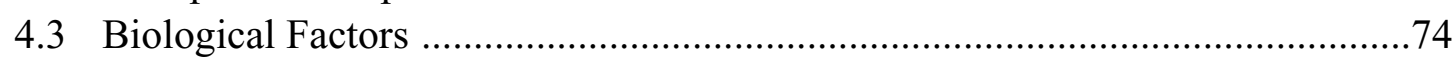

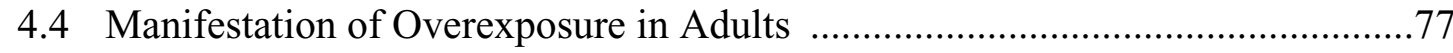

4.5 Recommendations for Effective/Equivalent Dose Limitations ...........................78

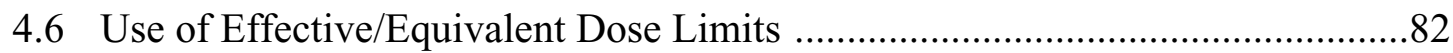

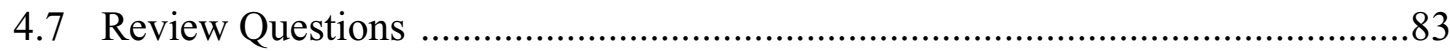

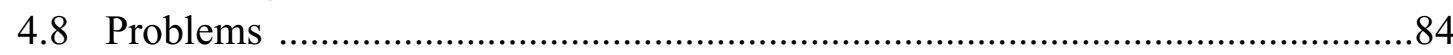

Chapter 5

Common Survey and Calibration Instruments $\quad 85$

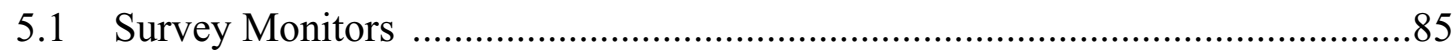

5.1.1 Characteristics of an Effective Survey Instrument .................................86

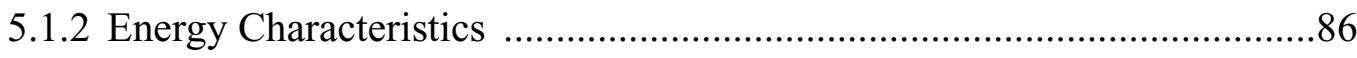

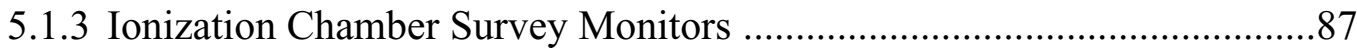

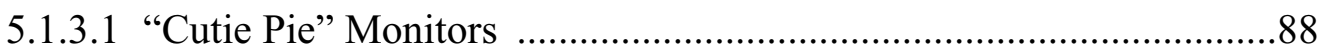

5.1.3.2 Digital Ionization Chamber Monitors ...............................................89

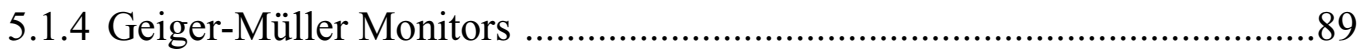




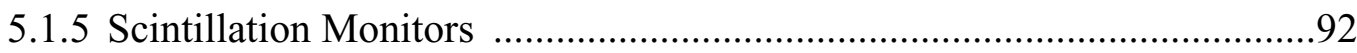

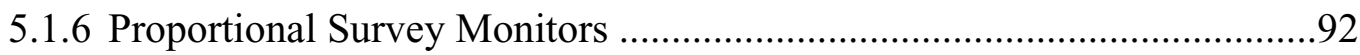

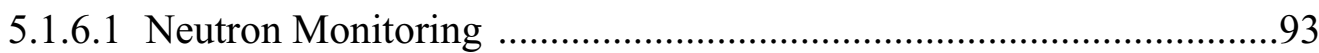

5.1.6.2 Low-Energy Particle Monitoring ……………..................................95

5.1.7 Survey Instruments for Container Monitoring

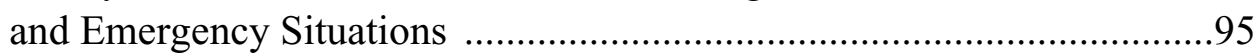

5.2 Calibration Instruments for Radiation Sources ...............................................97

5.2.1 Precision Electrometer/Dosemeter .......................................................97

5.2.2 Non-invasive Quality Assurance Test System .........................................98

5.2.3 Patient Air Kerma Monitors .............................................................100

5.2.4 Calibration of Radioactive Sources ........................................................101

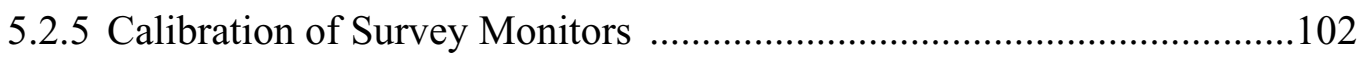

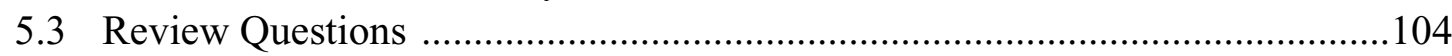

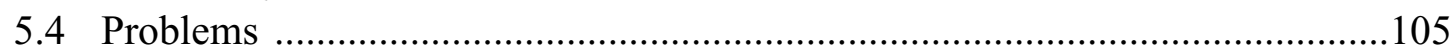

Chapter 6

$\begin{array}{lr}\text { Personnel Monitors } & 107\end{array}$

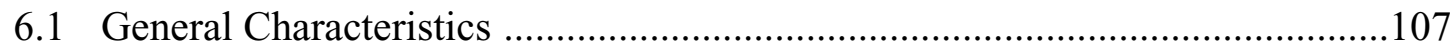

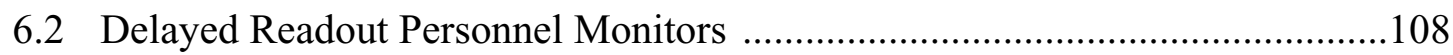

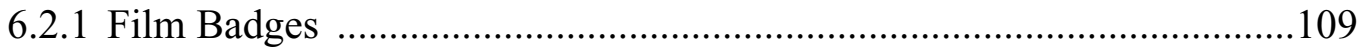

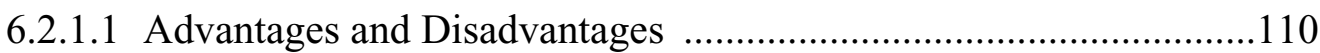

6.2.2 Thermoluminescent Dosimeters (TLDs) ............................................111

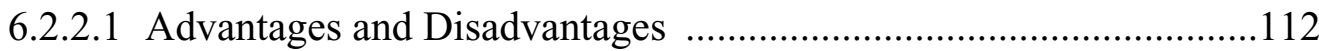

6.2.3 Optically Stimulated Luminescence (OSL) ...........................................112

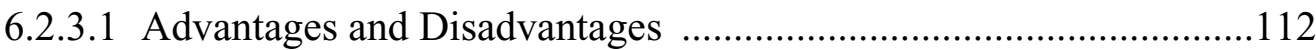

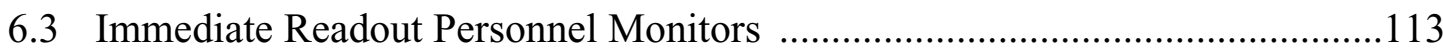

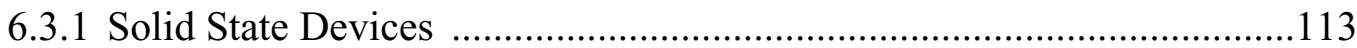

6.3.1.1 Advantages and Disadvantages ........................................................113

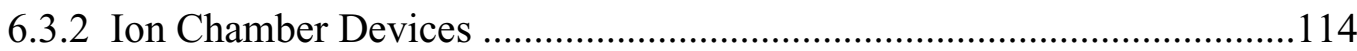

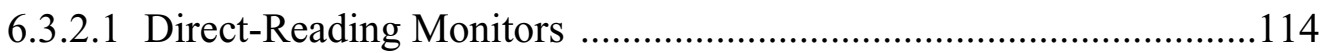

6.3.2.2 Condenser-Type Pocket Chambers ...............................................115

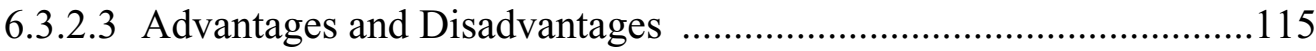

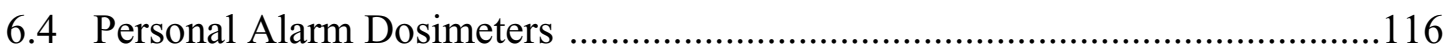

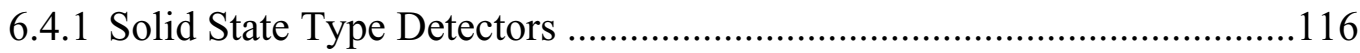

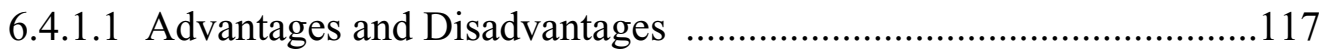

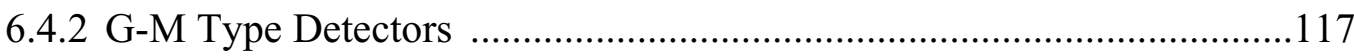

6.4.2.1 Advantages and Disadvantages ...................................................117

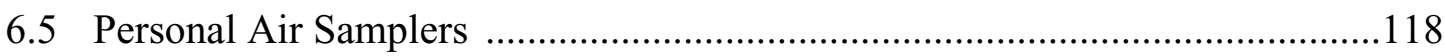

6.5.1 Advantages and Disadvantages ........................................................118

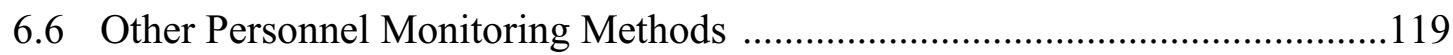

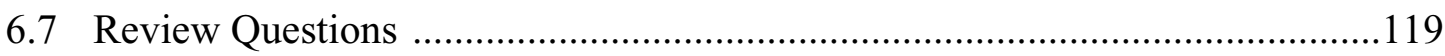

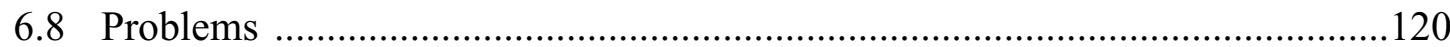


Chapter 7

Practical Means of Radiation Protection $\quad 121$

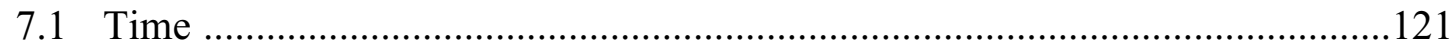

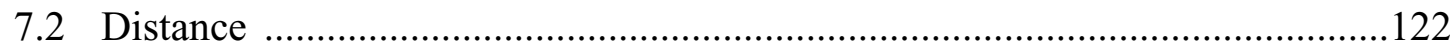

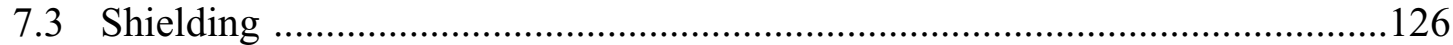

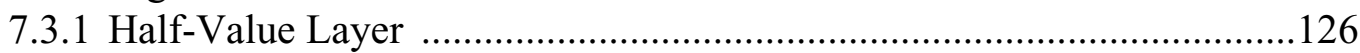

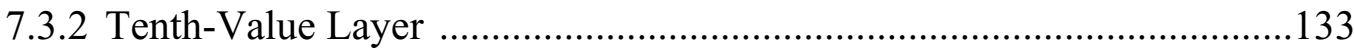

7.3.3 Polyenergetic Beams of Photons ............................................................134

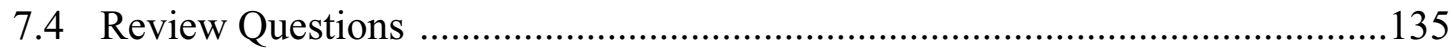

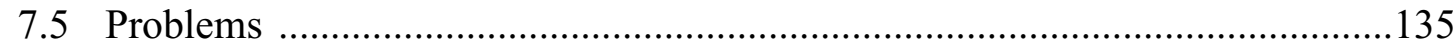

Chapter 8

Principles Governing Specific Devices: Good Working Habits 137

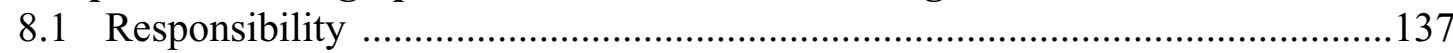

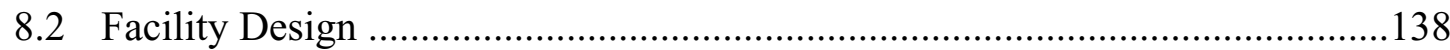

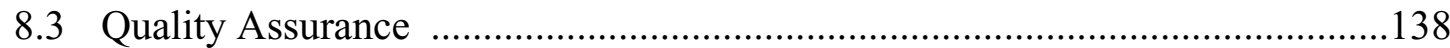

8.4 Specific Recommendations for X-Ray Generating Equipment .........................142

8.4.1 Leakage, Beam Size Definition, and Beam Filtration ............................142

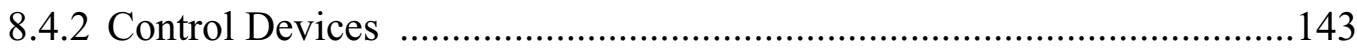

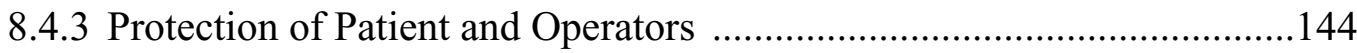

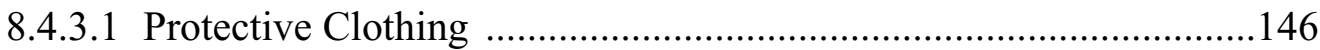

8.5 Specific Recommendations for Sealed Sources of Radioactivity .....................146

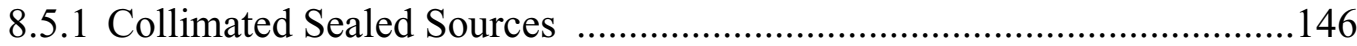

8.5.2 Noncollimated Sealed Sources ..........................................................147

8.6 Specific Recommendations for Particle Accelerators ......................................148

8.7 Specific Recommendations for Neutron Generators ........................................149

8.8 Specific Recommendations for Unsealed Sources of Radioactivity .................149

8.8.1 Common External Sources of Irradiation ..............................................150

8.8.2 Protection from External Sources of Irradiation .....................................150

8.8.3 Common Internal Sources of Irradiation ...............................................152

8.8.4 Common Methods of Acquiring Internal Irradiation Sources ................154

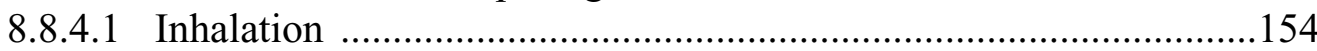

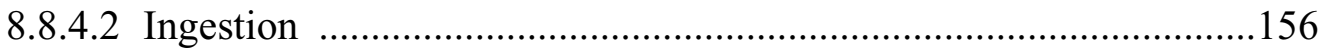

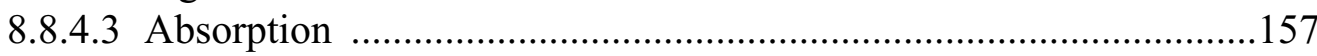

8.8.5 Protection from Internal Sources of Irradiation ....................................157

8.8.6 Specific Contamination Control Devices ...................................................159

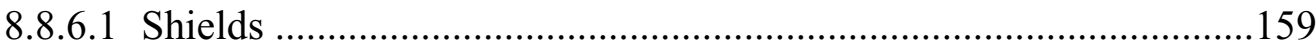

8.8.6.2 Remote Handling Equipment .....................................................160

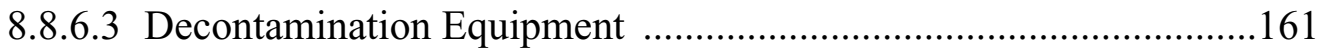

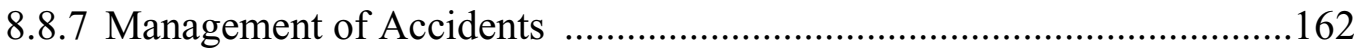

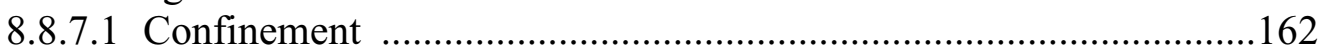

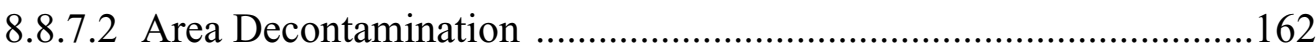

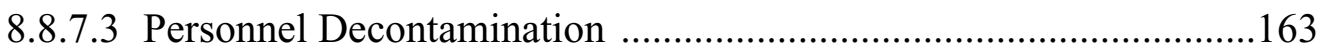

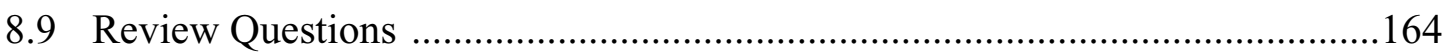

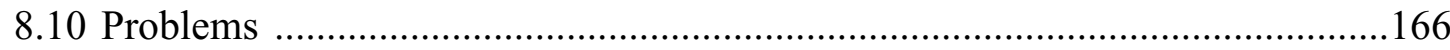


Chapter 9

Radionuclides and the Law $\quad 167$

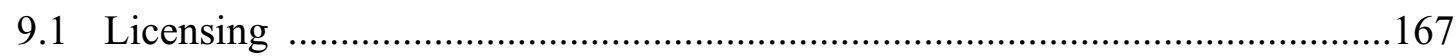

9.1.1 Nuclear Regulatory Commission .....................................................168

9.1.2 Radiation Control for Health and Safety Act of 1968 ...........................168

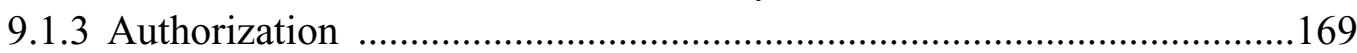

9.2 Record Keeping ............................................................................... 171

9.3 Area Posting and Radionuclide Labeling ................................................. 172

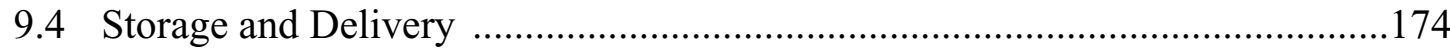

9.5 Transport ........................................................................................ 175

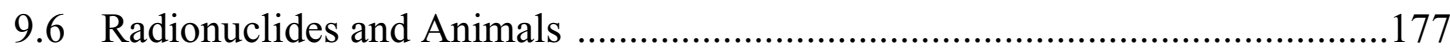

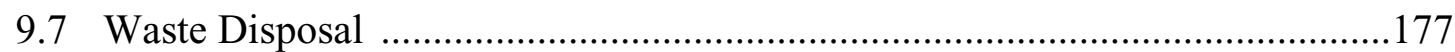

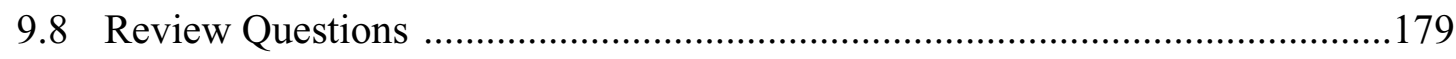

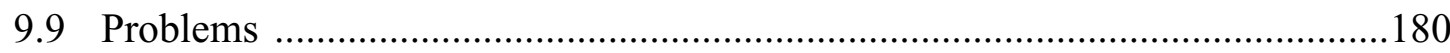

Chapter 10

$\begin{array}{lc}\text { Shielding from External Radiation } & 181\end{array}$

10.1 Protection from External Unsealed $\gamma$-Ray Photons .......................................182

10.2 Protection from Sources of X-Ray and Annihilation Photons .......................186

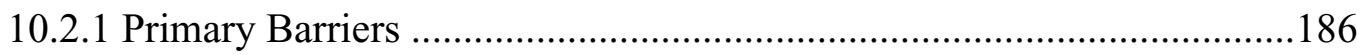

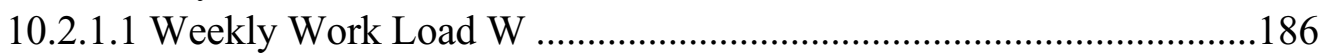

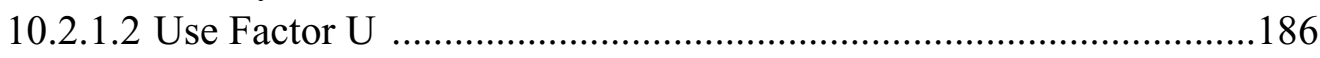

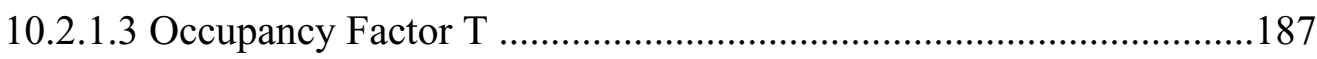

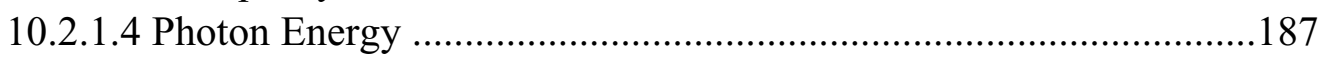

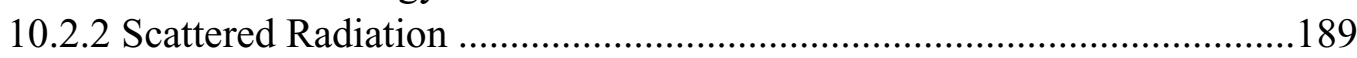

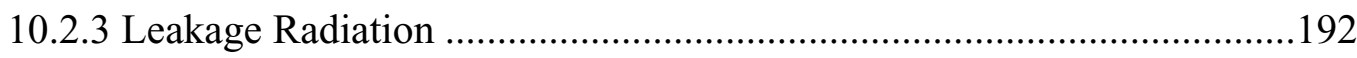

10.2.3.1 Scattered Radiation from CT Units ...........................................195

10.2.4 Special Considerations for PET Units ................................................197

10.2.5 Barriers Against Neutron Generation by Linear Accelerators ...............197

10.2.6 Special Recommendations for Dental and Veterinary Units .................198

10.3 Barriers for Sealed-Sources .................................................................. 198

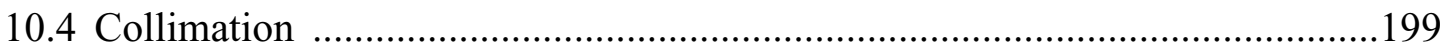

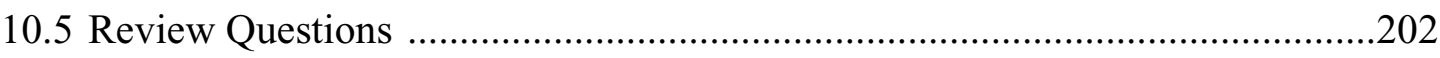

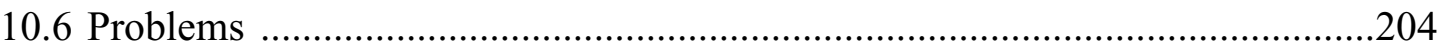

Chapter 11

$\begin{array}{ll}\text { Internal Dosimetry } & \mathbf{2 0 5}\end{array}$

11.1 Mean Absorbed Dose ............................................................................205

11.2 Effective Half-Life .................................................................................211

11.3 Accumulated Activity and Residence Time ..............................................213

11.4 Use of the MIRD Tables ........................................................................219

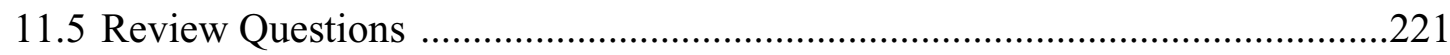

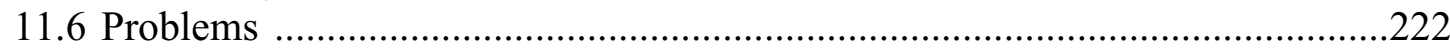


Chapter 12

Absorbed Dose from External Photons $\quad 223$

12.1 Kerma and Absorbed Dose in Air ...........................................................223

12.2 Absorbed Dose to a Small Mass of Tissue Exposed in Free Space ................224

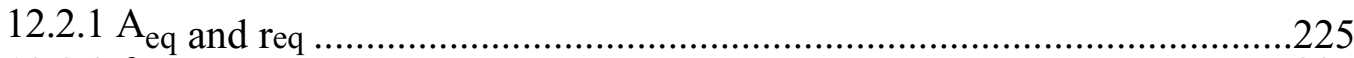

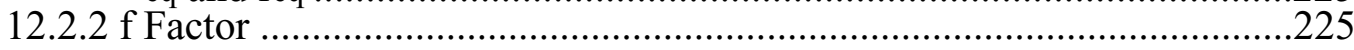

12.3 The Bragg-Gray Principle ........................................................................226

12.4 Other Absorbed Dose Calculation Methods .................................................230

12.5 Absorbed Dose in Selected Diagnostic X-Ray Procedures .............................230

12.6 Conclusions ..........................................................................................23

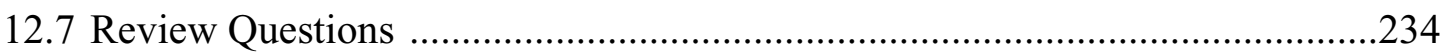

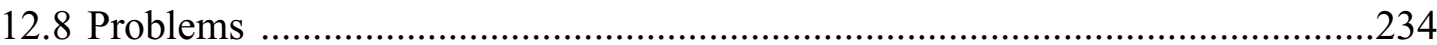

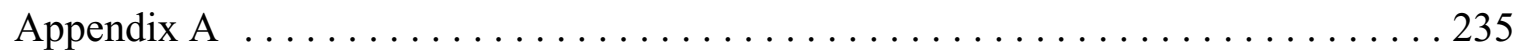

The Units of Physics $\quad 235$

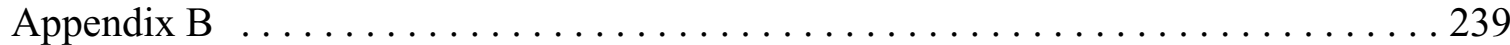

$\begin{array}{ll}\text { The Roentgen and the Rad } & 239\end{array}$

B.1 The Roentgen ....................................................................................239

B.2 The Rad .............................................................................................2239

B.3 Equivalence between Roentgen and $\mathrm{Rad}$..................................................240

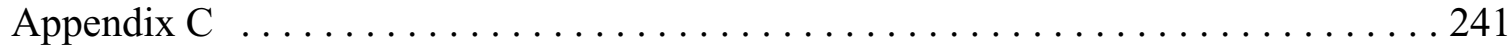

Logarithms

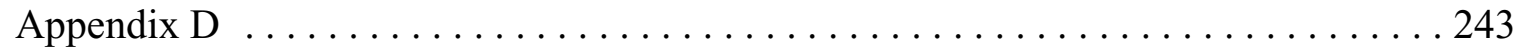

Greek Alphabet $\quad 243$

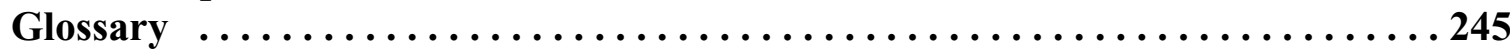

References .................................... 281

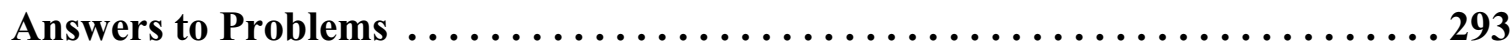

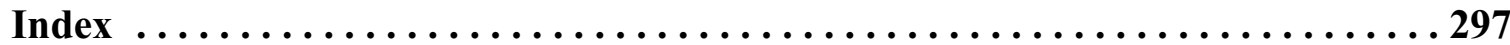

